\title{
METHYLPREDNISOLONE DOES NOT BENEFIT PATIENTS UNDERGOING CORONARY ARTERY BYPASS GRAFTING AND EARLY TRACHEAL EXTUBATION
}

Mark A. Chaney, MDa

Ramòn A. Durazo-Arvizu, $\mathrm{PhD}^{\mathrm{b}}$

Mihail P. Nikolov, MD $^{\mathrm{c}}$

Bradford P. Blakeman, MD $^{\mathrm{d}}$

Mamdouh Bakhos, MD $^{\mathrm{d}}$
Objective: We sought to determine whether methylprednisolone, when administered to patients undergoing cardiac surgery, is able to ward off the detrimental hemodynamic and pulmonary alterations associated with cardiopulmonary bypass.

Methods: After institutional review board approval and informed consent was obtained, 90 patients scheduled for elective cardiac surgery were randomized to 1 of 3 groups. Group 30MP patients received $30 \mathrm{mg} / \mathrm{kg}$ intravenous methylprednisolone during sternotomy and $30 \mathrm{mg} / \mathrm{kg}$ during initiation of cardiopulmonary bypass, group $15 \mathrm{MP}$ patients received $15 \mathrm{mg} / \mathrm{kg}$ methylprednisolone at the same 2 times, and group NS patients received similar volumes of isotonic sodium chloride solution at the same 2 times. Perioperative care was standardized, and all caregivers were blinded to treatment group. Various hemodynamic and pulmonary measurements were obtained perioperatively, as well as fluid balance, weight, peak postoperative blood glucose level, and tracheal extubation time.

Results: Demographic and clinical characteristics of patients and intraoperative data were similar among the 3 groups. Patients receiving methylprednisolone (either dose) exhibited significantly increased cardiac index $(P=.0006)$, significantly decreased systemic vascular resistance $(P=.0005)$, and significantly increased shunt flow $(P=.0020)$ during the immediate postoperative period. All 3 groups exhibited significant increases in alveolararterial oxygen gradient $(P<.0001)$, significant decreases in dynamic lung compliance $(P<.0001)$, and significant decreases in static lung compliance $(P<.0001)$ during the immediate postoperative period, with no differences between groups. Perioperative fluid balance and weights were similar between groups. A statistically significant difference in peak postoperative blood glucose level existed $(P=.016)$ among group NS $(234 \pm 96 \mathrm{mg} / \mathrm{dL})$, group 15MP $(292 \pm 93 \mathrm{mg} / \mathrm{dL})$, and group 30MP $(311 \pm 90 \mathrm{mg} / \mathrm{dL})$. In patients extubated within 12 hours of intensive care unit arrival, a statistically significant difference in extubation times existed $(P=.025)$ between group NS $(5.7 \pm 2.3$ hours $)$, group $15 \mathrm{MP}(5.9 \pm 2.2$ hours $)$, and group 30MP $(7.5 \pm 2.7$ hours).

Conclusions: Methylprednisolone, as used in this investigation, offers no clinical benefits to patients undergoing elective coronary artery bypass grafting with cardiopulmonary bypass and may in fact be detrimental by initiating postoperative hyperglycemia and possibly hindering early postoperative tracheal extubation for undetermined reasons. (J Thorac Cardiovasc Surg 2001;121:561-9)
From the Department of Anesthesia and Critical Care, University of Chicago, ${ }^{\text {a }}$ Chicago, Ill, the Department of Preventative Medicine and Epidemiology, ${ }^{\text {b }}$ the Department of Thoracic and Cardiovascular Surgery, ${ }^{\mathrm{d}}$ Loyola University Medical Center, Maywood, Ill, and the Alexian Brothers Medical Center, ${ }^{\mathrm{c}}$ Elk Grove Village, Ill.
Supported by the Loyola University Medical Center, Department of Anesthesiology, Research Fund.

Copyright (ㄷ 2001 by The American Association for Thoracic Surgery

$0022-5223 / 2001 \$ 35.00+0 \quad \mathbf{1 2 / 1 / 1 1 2 3 4 3}$

doi: $10.1067 / \mathrm{mtc} .2001 .112343$ 
C ardiopulmonary bypass (CPB) exposes blood to large areas of synthetic materials that trigger the production and release of numerous chemotactic and vasoactive substances. ${ }^{1}$ This ensuing abnormal wholebody inflammatory response can complicate the postoperative period by causing major organ dysfunction. Traditionally, methylprednisolone has been administered to patients undergoing cardiac surgery to ward off these detrimental physiologic alterations, yet few wellcontrolled investigations exist, and use of the drug in this setting remains controversial. Our group was the first to rigorously examine use of methylprednisolone in patients undergoing coronary artery bypass grafting $(\mathrm{CABG})$ and early extubation. ${ }^{2,3}$ Somewhat surprisingly, we found that methylprednisolone ( $30 \mathrm{mg} / \mathrm{kg}$ during sternotomy and $30 \mathrm{mg} / \mathrm{kg}$ during initiation of CPB) caused larger increases in postoperative alveolar-arterial (A-a) oxygen gradient and shunt flow, was unable to prevent postoperative decreases in lung compliance, was associated with a higher likelihood of requiring postoperative hemodynamic support, and prolonged tracheal extubation times when compared with placebo controls. $^{2,3}$ These results indicate that use of the drug in this setting may hinder early tracheal extubation., ${ }^{2,3}$ This study was undertaken to determine whether these unexpected results could be duplicated and possibly ascertain why methylprednisolone was detrimental in this setting. Because larger doses of methylprednisolone may induce sodium and water retention, which may initiate pulmonary edema in susceptible patients, ${ }^{4}$ this investigation assessed perioperative fluid balance and weights in study patients along with standard pulmonary and hemodynamic parameters. Furthermore, 2 different doses of methylprednisolone (30 mg/kg twice and $15 \mathrm{mg} / \mathrm{kg}$ twice) are compared with placebo controls.

\section{Methods}

After institutional review board approval and informed consent were obtained, 90 patients scheduled for elective CABG and early extubation were studied. At our institution, we view all patients scheduled for elective CABG as candidates for early extubation, including those undergoing reoperations and those with decreased left ventricular function (ejection fraction $<40 \%$ ). Patients receiving preoperative steroids or who had undergone previous lung surgery were excluded from participation. Patients requiring preoperative intravenous inotropic or vasoactive drugs, intra-aortic balloon support, supplemental oxygen, or mechanical ventilation were excluded.

Each patient was randomized to 1 of 3 groups. Two groups received intravenous methylprednisolone sodium succinate (A-Methapred; Abbott Laboratories, North Chicago, Ill), and 1 group received intravenous isotonic sodium chloride solu- tion. Group 30MP patients received $30 \mathrm{mg} / \mathrm{kg}$ intravenous methylprednisolone during sternotomy and $30 \mathrm{mg} / \mathrm{kg}$ during initiation of CPB, group 15MP patients received $15 \mathrm{mg} / \mathrm{kg}$ of intravenous methylprednisolone at the same 2 times, and group NS patients received similar volumes of intravenous isotonic sodium chloride solution at the same 2 times. An anesthesia research nurse performed the randomization and prepared the 2 syringes of blinded solution that were administered by the anesthesiologist managing the case. All physicians and nursing staff caring for the patients perioperatively were unaware of treatment group.

The intraoperative anesthetic technique was standardized and consisted of intravenous fentanyl $(20 \mathrm{mg} / \mathrm{kg})$, midazolam (150 $\mathrm{mg} / \mathrm{kg}$ ), and vecuronium bromide. All of the fentanyl was administered before sternotomy. Regarding midazolam, approximately $70 \%$ of the calculated dose was administered before sternotomy, and the balance was administered during rewarming. If required, inhaled isoflurane, intravenous nitroglycerin, or both were used for blood pressure control before initiation of $\mathrm{CPB}$. Hypothermic $\mathrm{CPB}$ (to a lowest temperature of $26^{\circ} \mathrm{C}$ ) with a membrane oxygenator and crystalloid prime $(2.0 \mathrm{~L}$ of lactated Ringer's solution and $50 \mathrm{mEq}$ sodium bicarbonate) was used in all patients. Nonpulsatile flows were maintained between 2.4 and $2.8 \mathrm{~L} \cdot \mathrm{min}^{-1} \cdot \mathrm{m}^{-2}$, and if needed, isoflurane was used by the perfusionist to maintain perfusion pressure in the range of 50 to $70 \mathrm{~mm} \mathrm{Hg}$. Intermittent antegrade hypothermic crystalloid cardioplegia was used in all patients. Alpha-stat blood gas management was used in all patients. The lungs were allowed to deflate during CPB. Separation from CPB was facilitated with intravenous dobutamine, norepinephrine, nitroglycerin, or some combination thereof at the discretion of the anesthesiologist managing the case.

Hemodynamic and pulmonary measurements were obtained at 4 times: 10 minutes after intubation (time A), 10 minutes after sternotomy (time B), 10 minutes after sternal closure (time C), and 60 minutes after intensive care unit (ICU) arrival (time D). A pulmonary artery catheter (Swan-Ganz Thermodilution Paceport Catheter; Baxter Healthcare Corporation, Irvine, Calif) was used in all patients to facilitate data collection. Hemodynamic measurements included heart rate, mean arterial pressure, central venous pressure, mean pulmonary artery pressure, and pulmonary artery occlusive pressure. Cardiac outputs were obtained at end-expiration in triplicate and averaged. Cardiac index (CI), systemic vascular resistance (SVR), pulmonary vascular resistance (PVR), and shunt flow were calculated by using standard equations (Appendix I). Pulmonary measurements included A-a oxygen gradient, arterial carbon dioxide tension, dynamic lung compliance, and static lung compliance computed by standard equations (Appendix II). Mechanical ventilation parameters were standardized (respiratory rate, 8 breaths $/ \mathrm{min}$; tidal volume, $10 \mathrm{~mL} / \mathrm{kg}$; fraction of inspired oxygen $\left[\mathrm{FIO}_{2}\right], 1.0$; positive end-expiratory pressure, +5 $\mathrm{cm} \mathrm{H}_{2} \mathrm{O}$; and inspiratory/expiratory ratio, 1:3) for at least 10 minutes before each measurement. In each patient the inspiratory flow was adjusted so that the calculated tidal volume was delivered during the entire inspiratory cycle (creating the lowest peak airway pressure). 
Perioperative fluid balance and weights were recorded in all patients. Total operating room intravenous input (eg, crystalloid and blood products) and total operating room urine output, as well as total intravenous input (eg, crystalloid and blood products) and total output (urine and chest tube) during the initial 24 postoperative hours, were recorded. All patients were weighed 3 times: immediately before transfer to the operating room (baseline) and at 2 and 24 hours after ICU arrival. The same sling scale (Scale-Tronix 2001 Sling Scale; Scale-Tronix, Inc, Wheaton, Ill) was used for every weight measurement.

After completion of CABG, patients were transferred to the ICU, where postoperative care was standardized and tracheal extubation was accomplished at the earliest clinically appropriate time. Criteria for extubation in our ICU include an appropriate sensorium, normothermia, hemodynamic stability, adequate pulmonary function (arterial partial pressure of oxygen, $>60 \mathrm{~mm} \mathrm{Hg}$ with an $\mathrm{FIO}_{2}$ of 0.4 ), adequate urine output, and minimal chest tube output. If a patient had hypertension, tachycardia, and/or excessive movement at a time when tracheal extubation was not yet appropriate (for any reason), the ICU nurse was free to administer small amounts of intravenous midazolam. In patients who were not extubated within 24 hours of ICU arrival, the reason for prolonged intubation (eg, hemodynamic instability and oxygenation difficulties) was ascertained.

Postoperative complications and treatments were recorded daily until hospital discharge. All patients had a creatine kinase (CK) level assayed (by means of coupled enzymatic reactions on the basis of Rosalki's modification of the Oliver procedure) and an electrocardiogram recorded at 4 AM on the first postoperative day. If the total CK level was greater than $200 \mathrm{IU} / \mathrm{L}$, CK-MB levels were assayed (by using the monoclonal antibody technique), and the CK-MB index was calculated (CK$\mathrm{MB} /$ total $\mathrm{CK} \times 100$ ). If the initial total $\mathrm{CK}$ was greater than 200 IU/L, 2 additional total CK and CK-MB levels were measured 6 and 12 hours later. Sensitivity of the CK-MB assay in our laboratory is $0.4 \mathrm{ng} / \mathrm{mL}$. Perioperative myocardial infarction was defined as a postoperative CK-MB index of greater than 3.0, postoperative electrocardiographic evidence (new Q waves or ST segment elevation) of infarction, or both.

The Pearson $\chi^{2}$ or Fisher exact tests were applied to categoric data. One-way analysis of variance (ANOVA) was used to test the difference between means in the 3 groups regarding demographic and clinical characteristics of patients and appropriate perioperative data. To account for repeated measurements of perioperative hemodynamic and pulmonary data, repeated-measures ANOVA was used, along with statistical construct, to compare mean measurements 10 minutes after intubation and 60 minutes after ICU arrival (with and without the Bonferroni correction). Results are expressed as the mean \pm 1 SD or, when indicated in the appropriate table, the number of patients, unless otherwise indicated.

\section{Results}

Thirty patients were randomized to each group. One patient in group NS and 1 patient in group 15MP were omitted from statistical analysis because of deviation
Table I. Demographic and clinical characteristics of patients

\begin{tabular}{lccc}
\hline & Group & Group & Group \\
& $N S$ & $15 M P$ & $30 M P$ \\
$(n=29)$ & $(n=29)$ & $(n=30)$ \\
\hline Preoperative history & & & \\
Age, y (range) & $67 \pm 10$ & $62 \pm 12$ & $67 \pm 9$ \\
& $(43-85)$ & $(37-81)$ & $(54-81)$ \\
Male & 22 & 24 & 22 \\
Female & 7 & 5 & 8 \\
Height (cm) & $172 \pm 8$ & $173 \pm 9$ & $169 \pm 11$ \\
Weight (kg) & $85 \pm 20$ & $84 \pm 15$ & $79 \pm 16$ \\
Smoking history (packs/y) & & & \\
10-20 & 9 & 5 & 7 \\
21-40 & 3 & 6 & 5 \\
41-60 & 3 & 5 & 7 \\
$>$ 60 & 5 & 3 & 5 \\
Actively smoking & 5 & 3 & 5 \\
Never smoked & 9 & 10 & 6 \\
Preoperative medications & & & \\
B-Blocker & 14 & 18 & 18 \\
Calcium channel blocker & 5 & 3 & 7 \\
Nitrate & 12 & 16 & 15 \\
ACE inhibitor & 5 & 12 & 12 \\
Digitalis preparation & 4 & 2 & 7 \\
Diuretic & 7 & 4 & 7 \\
Oral hypoglycemic & 8 & 6 & 5 \\
Insulin & 3 & 3 & 5 \\
Inhaled bronchodilators & 1 & 2 & 1 \\
None & 2 & 1 & 0 \\
\hline
\end{tabular}

$A C E$, Angiotensin-converting enzyme.

from the study protocol. Demographic and clinical characteristics of patients and intraoperative data are presented in Tables I and II, respectively. Statistical analysis with the Pearson $\chi^{2}$ and Fisher exact test and 1-way ANOVA revealed no differences between groups regarding demographic and clinical characteristics and intraoperative data.

Perioperative hemodynamic data are presented in Tables III and IV. Regarding Table III, repeated-measures ANOVA revealed that differences over time (times $\mathrm{A}, \mathrm{B}, \mathrm{C}$, and $\mathrm{D}$ ) within groups in heart rate $(P<.0001)$, mean arterial pressure $(P<.0001)$, central venous pressure $(P<.0001)$, mean pulmonary artery pressure $(P<.0001)$, and pulmonary artery occlusive pressure $(P<.0001)$ were statistically significant, yet there were no differences between groups. Regarding Table IV, repeated-measures ANOVA revealed that differences over time (times A, B, C, and D) within groups in CI $(P=.0001)$, SVR $(P=.0098)$, PVR $(P=.0049)$, and shunt flow $(P<.0001)$ were statistically significant. When mean measurements at time A and time $\mathrm{D}$ were compared by statistically constructed repeated-measures ANOVA, differences in CI 
Table II. Intraoperative data

\begin{tabular}{|c|c|c|c|}
\hline & Group NS $(n=29)$ & Group $15 M P(n=29)$ & Group $30 M P(n=30)$ \\
\hline \multicolumn{4}{|l|}{ Anesthetic drugs } \\
\hline Fentanyl $(\mu \mathrm{g} / \mathrm{kg})$ & $20 \pm 0.3$ & $20 \pm 0.3$ & $20 \pm 0.4$ \\
\hline Midazolam ( $\mu \mathrm{g} / \mathrm{kg})$ & $150 \pm 39$ & $150 \pm 21$ & $139 \pm 23$ \\
\hline Vecuronium $(\mu \mathrm{g} / \mathrm{kg})$ & $431 \pm 186$ & $468 \pm 167$ & $436 \pm 182$ \\
\hline \multicolumn{4}{|l|}{ Before CPB } \\
\hline Isoflurane use & 29 & 26 & 29 \\
\hline Nitroglycerin use & 6 & 8 & 9 \\
\hline \multicolumn{4}{|l|}{ During CPB } \\
\hline Lowest temperature $\left({ }^{\circ} \mathrm{C}\right)$ & $28.8 \pm 1.8$ & $27.9 \pm 1.6$ & $27.9 \pm 1.8$ \\
\hline Highest ACT (s) & $608 \pm 141$ & $653 \pm 181$ & $633 \pm 129$ \\
\hline Total heparin (mg) & $295 \pm 93$ & $379 \pm 99$ & $311 \pm 120$ \\
\hline Total cardioplegia $(\mathrm{mL})$ & $3176 \pm 1086$ & $3034 \pm 932$ & $3309 \pm 991$ \\
\hline Countershocks required & 14 & 16 & 12 \\
\hline Crossclamp time (min) & $84 \pm 18$ & $82 \pm 29$ & $83 \pm 17$ \\
\hline $\mathrm{CPB}$ time (min) & $108 \pm 25$ & $107 \pm 30$ & $102 \pm 22$ \\
\hline \multicolumn{4}{|l|}{ After CPB } \\
\hline Dobutamine use & 20 & 23 & 20 \\
\hline Norepinephrine use & 3 & 4 & 6 \\
\hline Nitroglycerin use & 1 & 2 & 2 \\
\hline Isoflurane use & 6 & 6 & 5 \\
\hline Total protamine (mg) & $310 \pm 84$ & $348 \pm 88$ & $315 \pm 87$ \\
\hline \multicolumn{4}{|l|}{ Surgical data } \\
\hline Saphenous vein grafts (range) & $1-4$ & $0-5$ & $1-4$ \\
\hline LITA & 26 & 26 & 24 \\
\hline
\end{tabular}

$A C T$, Activated clotting time; LITA, left internal thoracic artery.

Table III. Perioperative hemodynamic data

\begin{tabular}{|c|c|c|c|c|}
\hline & Time A & Time B & Time $C$ & Time $D$ \\
\hline \multicolumn{5}{|c|}{ Heart rate (beats/min) } \\
\hline Group NS & $58.9 \pm 10.7$ & $62.6 \pm 12.6$ & $91.9 \pm 16.5$ & $89.2 \pm 12.5$ \\
\hline Group 15MP & $58.4 \pm 10.2$ & $61.0 \pm 13.8$ & $90.5 \pm 15.3$ & $86.0 \pm 16.0$ \\
\hline Group 30MP & $59.9 \pm 13.1$ & $68.0 \pm 13.4$ & $90.0 \pm 15.0$ & $87.0 \pm 15.7$ \\
\hline \multicolumn{5}{|c|}{ Mean arterial pressure $(\mathrm{mm} \mathrm{Hg})$} \\
\hline Group NS & $68.4 \pm 11.6$ & $79.6 \pm 10.9$ & $81.0 \pm 10.1$ & $80.9 \pm 13.8$ \\
\hline Group 15MP & $71.9 \pm 9.7$ & $82.1 \pm 12.6$ & $77.4 \pm 8.9$ & $78.3 \pm 14.4$ \\
\hline Group 30MP & $73.3 \pm 7.1$ & $79.3 \pm 10.8$ & $76.3 \pm 12.2$ & $73.2 \pm 12.2$ \\
\hline \multicolumn{5}{|c|}{ Central venous pressure $(\mathrm{mm} \mathrm{Hg})$} \\
\hline Group NS & $13.2 \pm 3.3$ & $12.8 \pm 4.8$ & $13.5 \pm 4.3$ & $8.9 \pm 3.7$ \\
\hline Group 15MP & $13.5 \pm 3.4$ & $14.0 \pm 3.4$ & $14.4 \pm 3.7$ & $8.4 \pm 2.6$ \\
\hline Group 30MP & $12.2 \pm 2.8$ & $13.1 \pm 3.6$ & $14.3 \pm 3.9$ & $8.2 \pm 4.1$ \\
\hline \multicolumn{5}{|c|}{ Mean pulmonary artery pressure $(\mathrm{mm} \mathrm{Hg})$} \\
\hline Group NS & $22.5 \pm 4.8$ & $22.6 \pm 5.3$ & $22.2 \pm 5.0$ & $18.7 \pm 4.2$ \\
\hline Group 15MP & $22.8 \pm 7.6$ & $23.4 \pm 6.0$ & $24.1 \pm 4.4$ & $18.8 \pm 5.3$ \\
\hline Group 30MP & $21.0 \pm 4.9$ & $24.1 \pm 7.5$ & $24.3 \pm 6.0$ & $18.6 \pm 4.4$ \\
\hline \multicolumn{5}{|c|}{ Pulmonary artery occlusive pressure (mm Hg) } \\
\hline Group NS & $15.7 \pm 4.1$ & $15.5 \pm 5.4$ & $8.1 \pm 5.7$ & $11.3 \pm 3.9$ \\
\hline Group 15MP & $14.8 \pm 3.4$ & $15.6 \pm 3.3$ & $7.8 \pm 4.2$ & $10.4 \pm 3.0$ \\
\hline Group 30MP & $14.5 \pm 3.6$ & $15.0 \pm 4.4$ & $9.5 \pm 5.3$ & $11.1 \pm 3.8$ \\
\hline
\end{tabular}

Time A, 10 minutes after intubation; Time B, 10 minutes after sternotomy; Time $C, 10$ minutes after sternal closure; Time D, 60 minutes after ICU arrival.

$(P=.0006)$, SVR $(P=.0005)$, and shunt flow $(P=.0020)$ were statistically significant. Further statistical analysis of data at time $\mathrm{A}$ and time $\mathrm{D}$ by using the Bonferroni correction revealed significant differences between group NS and group 30MP regarding CI
$(P<.0001)$, SVR $(P<.0001)$, and shunt flow $(P=.0030)$ and between group 15MP and group 30MP regarding shunt flow $(P=.0260)$. There were no differences among group NS, group 15MP, and group $30 \mathrm{MP}$ in the number of patients requiring dobutamine 
Table IV. Perioperative hemodynamic data

\begin{tabular}{|c|c|c|c|c|}
\hline & Time A & Time B & Time $C$ & Time D \\
\hline \multicolumn{5}{|c|}{$\mathrm{CI}\left(\mathrm{L} \cdot \mathrm{min}^{-1} \cdot \mathrm{m}^{-2}\right)$} \\
\hline Group NS & $2.4 \pm 0.7$ & $2.5 \pm 0.7$ & $2.7 \pm 1.0$ & $2.6 \pm 0.7$ \\
\hline Group 15MP & $2.4 \pm 0.6$ & $2.6 \pm 0.7$ & $3.2 \pm 0.9$ & $3.1 \pm 0.8$ \\
\hline Group 30MP & $2.3 \pm 0.4$ & $2.5 \pm 0.7$ & $3.4 \pm 1.2$ & $3.4 \pm 1.0^{*}$ \\
\hline \multicolumn{5}{|c|}{ SVR (dynes $\cdot \mathrm{s}^{-1} \cdot \mathrm{cm}^{-5}$ ) } \\
\hline Group NS & $1027 \pm 396$ & $1225 \pm 452$ & $1181 \pm 564$ & $1260 \pm 550$ \\
\hline Group 15MP & $1027 \pm 283$ & $1171 \pm 382$ & $875 \pm 316$ & $968 \pm 324$ \\
\hline Group 30MP & $1181 \pm 345$ & $1201 \pm 388$ & $883 \pm 406$ & $913 \pm 470 *$ \\
\hline \multicolumn{5}{|c|}{ PVR (dynes $\cdot \mathrm{s}^{-1} \cdot \mathrm{cm}^{-5}$ ) } \\
\hline Group NS & $125 \pm 47$ & $133 \pm 56$ & $252 \pm 147$ & $124 \pm 67$ \\
\hline Group 15MP & $139 \pm 101$ & $141 \pm 111$ & $232 \pm 102$ & $118 \pm 67$ \\
\hline Group 30MP & $123 \pm 61$ & $168 \pm 121$ & $213 \pm 129$ & $96 \pm 46$ \\
\hline \multicolumn{5}{|l|}{ Shunt flow (\%) } \\
\hline Group NS & $15.9 \pm 6.1$ & $16.0 \pm 6.0$ & $21.6 \pm 9.1$ & $23.1 \pm 7.7$ \\
\hline Group 15MP & $15.3 \pm 7.6$ & $14.1 \pm 5.4$ & $21.4 \pm 9.7$ & $24.1 \pm 8.5$ \\
\hline Group 30MP & $14.9 \pm 6.9$ & $16.8 \pm 6.6$ & $27.3 \pm 9.6$ & $30.1 \pm 8.8 \dagger$ \\
\hline
\end{tabular}

Time A, 10 minutes after intubation; Time B, 10 minutes after sternotomy; Time C, 10 minutes after sternal closure; Time D, 60 minutes after ICU arrival.

$* P<.0001$, group $30 \mathrm{MP}$ versus group NS.

$\dagger P=.0030$, group 30MP versus group NS

$\ddagger P=.0260$, group $30 \mathrm{MP}$ versus group $15 \mathrm{MP}$.

$(10,12$, and 15 , respectively), norepinephrine $(8,9$, and 9 , respectively), or nitroglycerin $(8,3$, and 7 , respectively) during the initial 24 hours of ICU stay.

Perioperative pulmonary data are presented in Table V. Repeated-measures ANOVA revealed that differences over time (times A, B, C, and D) within groups in A-a oxygen gradient $(P<.0001)$, arterial carbon dioxide tension $(P<.0001)$, dynamic lung compliance $(P<.0001)$, and static lung compliance $(P<.0001)$ were statistically significant, yet there were no differences between groups.

Perioperative fluid balance and weights are presented in Table VI. One-way ANOVA revealed no differences between groups regarding perioperative fluid balance. All 3 groups experienced equivalent significant $(P<$ .0001) postoperative increases in weight.

Extubation times are presented in Table VII. When all extubated patients and patients extubated within 24 hours of ICU arrival were analyzed, there was no statistically significant difference between groups despite a trend toward prolonged extubation times in patients receiving methylprednisolone. However, when patients extubated within 12 hours of ICU arrival were analyzed, 1-way ANOVA revealed a statistically significant difference in extubation times $(P=.025)$ between groups, and Bonferroni correction comparing group NS with group 30MP yielded a $P$ value of .038 . Midazolam administration from arrival in the ICU to extubation was similar among group NS $(5.4 \pm 7.2$ $\mathrm{mg})$, group 15MP $(3.9 \pm 4.7 \mathrm{mg})$, and group 30MP (2.9 $\pm 5.6 \mathrm{mg}$ ). More patients in group 30MP (14) required no midazolam before extubation than in group $15 \mathrm{MP}$ (11) and group NS (7).

During the initial 24 ICU hours, 1-way ANOVA revealed a statistically significant difference in peak postoperative blood glucose level $(P=.016)$ among patients in group NS $(234 \pm 96 \mathrm{mg} / \mathrm{dL})$, group 15MP $(292 \pm 93 \mathrm{mg} / \mathrm{dL})$, and group 30MP $(311 \pm 90 \mathrm{mg} / \mathrm{dL})$. Postoperative atrial fibrillation occurred in 9 group NS patients, 8 group 15MP patients, and 9 group 30MP patients. One patient in group NS had a perioperative myocardial infarction (electrocardiographic changes) yet otherwise had an uneventful postoperative course. One patient in group NS and 1 patient in group 30MP had a postoperative cerebral infarction. Both patients were eventually discharged to a chronic care facility. One patient in group NS died. This patient was initially extubated uneventfully yet became asystolic late on the first postoperative day. Re-exploration revealed dysfunctional saphenous vein grafts, and the patient died on the fifth postoperative day. Of the 88 patients, 87 were eventually discharged from the hospital. The mean duration of postoperative hospital stay among patients in group NS ( $6.5 \pm 4.6$ days), group $15 \mathrm{MP}(6.0 \pm 4.8$ days $)$, and group $30 \mathrm{MP}(6.7 \pm 3.4$ days $)$ was similar.

\section{Discussion}

This prospective randomized double-blind placebocontrolled clinical study reveals that administration of methylprednisolone to patients undergoing elective 
Table V. Perioperative pulmonary data

\begin{tabular}{|c|c|c|c|c|}
\hline & Time A & Time $B$ & Time $C$ & Time $D$ \\
\hline \multicolumn{5}{|c|}{ A-a oxygen gradient $(\mathrm{mm} \mathrm{Hg})$} \\
\hline Group NS & $254.2 \pm 95.1$ & $246.7 \pm 91.4$ & $352.3 \pm 123.0$ & $393.3 \pm 83.7$ \\
\hline Group 15MP & $219.3 \pm 111.9$ & $219.4 \pm 96.1$ & $318.8 \pm 127.4$ & $364.1 \pm 106.0$ \\
\hline Group 30MP & $233.7 \pm 119.6$ & $243.7 \pm 128.9$ & $373.6 \pm 145.5$ & $413.7 \pm 126.5$ \\
\hline \multicolumn{5}{|c|}{ Arterial carbon dioxide tension $(\mathrm{mm} \mathrm{Hg})$} \\
\hline Group NS & $35.9 \pm 4.4$ & $34.2 \pm 5.9$ & $38.0 \pm 4.9$ & $45.2 \pm 7.7$ \\
\hline Group 15MP & $37.5 \pm 4.2$ & $35.1 \pm 4.3$ & $39.8 \pm 4.9$ & $46.2 \pm 6.7$ \\
\hline Group 30MP & $36.8 \pm 3.6$ & $35.4 \pm 4.1$ & $40.4 \pm 4.5$ & $45.5 \pm 6.4$ \\
\hline \multicolumn{5}{|c|}{ Dynamic lung compliance $\left(\mathrm{mL} / \mathrm{cm} \mathrm{H}_{2} \mathrm{O}\right)$} \\
\hline Group NS & $49.4 \pm 14.3$ & $47.7 \pm 12.8$ & $43.2 \pm 12.2$ & $33.1 \pm 8.5$ \\
\hline Group 15MP & $48.3 \pm 10.5$ & $47.0 \pm 9.2$ & $41.4 \pm 8.3$ & $36.5 \pm 6.1$ \\
\hline Group 30MP & $48.1 \pm 12.6$ & $47.7 \pm 12.9$ & $41.7 \pm 10.1$ & $35.7 \pm 6.4$ \\
\hline \multicolumn{5}{|c|}{ Static lung compliance $\left(\mathrm{mL} / \mathrm{cm} \mathrm{H}_{2} \mathrm{O}\right)$} \\
\hline Group NS & $71.4 \pm 27.7$ & $68.2 \pm 28.7$ & $61.5 \pm 26.0$ & $49.3 \pm 13.1$ \\
\hline Group 15MP & $69.4 \pm 41.0$ & $61.6 \pm 17.3$ & $55.5 \pm 24.1$ & $57.0 \pm 13.9$ \\
\hline Group 30MP & $73.6 \pm 44.6$ & $75.4 \pm 59.4$ & $63.2 \pm 38.3$ & $54.8 \pm 13.3$ \\
\hline
\end{tabular}

Time A, 10 minutes after intubation; Time B, 10 minutes after sternotomy; Time $C, 10$ minutes after sternal closure; Time D, 60 minutes after ICU arrival.

Table VI. Perioperative fluid balance and weights

\begin{tabular}{|c|c|c|c|}
\hline & Group NS $(n=29)$ & Group $15 M P(n=29)$ & Group $30 M P(n=30)$ \\
\hline Total OR IV input (mL) & $2660 \pm 505$ & $3096 \pm 1739$ & $3097 \pm 964$ \\
\hline Total OR urine output (mL) & $844 \pm 564$ & $976 \pm 770$ & $819 \pm 583$ \\
\hline Initial 24-h ICU total IV input (mL) & $3714 \pm 1450$ & $3671 \pm 1677$ & $3491 \pm 1432$ \\
\hline Initial 24-h ICU total output (mL) & $3281 \pm 1004$ & $3668 \pm 1301$ & $3491 \pm 1193$ \\
\hline Baseline weight $(\mathrm{kg})$ & $85.5 \pm 20.3$ & $84.0 \pm 14.9$ & $78.9 \pm 16.4$ \\
\hline Weight $2 \mathrm{~h}$ after ICU arrival (kg) & $90.7 \pm 18.4 *$ & $90.9 \pm 15.3^{*}$ & $85.6 \pm 16.8^{*}$ \\
\hline Weight $24 \mathrm{~h}$ after ICU arrival (kg) & $91.3 \pm 20.3^{*}$ & $88.7 \pm 15.7^{*}$ & $84.6 \pm 17.1^{*}$ \\
\hline
\end{tabular}

$O R$, Operating room; $I V$, intravenous.

$* P<.0001$ when compared with baseline weight.

CABG with CPB increases postoperative CI, decreases postoperative SVR, increases postoperative shunt flow, is unable to prevent postoperative pulmonary dysfunction (increased A-a oxygen gradient and decreased dynamic and static lung compliance) or prevent postoperative weight gain, initiates postoperative hyperglycemia, and prolongs tracheal extubation time. Thus, methylprednisolone, as used in this investigation, offers no clinical benefits to patients undergoing elective CABG with CPB and may in fact be detrimental by initiating postoperative hyperglycemia and possibly hindering early postoperative tracheal extubation.

Patients undergoing cardiac surgery experience an abnormal whole-body inflammatory response after initiation of $\mathrm{CPB}$, which causes detrimental postoperative changes in pulmonary function and hemodynamics. ${ }^{1}$ Inhibition of detrimental CPB-induced physiologic effects may be possible with corticosteroids. In the 1960s, methylprednisolone became the drug of choice because of its clinical efficacy in shock and sepsis and its advantageous side effect profile. ${ }^{5}$ The dose used, 30 $\mathrm{mg} / \mathrm{kg}$, was empirically chosen and remains the standard. However, a supplemental dose must be administered at initiation of CPB to maintain adequate plasma levels of drug into the postoperative period. ${ }^{6}$ Although many studies have investigated use of methylprednisolone in preventing detrimental CPB-induced physiologic effects, it is still unclear whether the drug truly is beneficial in this setting. ${ }^{7-32}$

Whether administration of methylprednisolone before CPB enhances postoperative hemodynamic stability remains controversial." The observed hemodynamic changes in this investigation regarding CI, SVR, and shunt flow were similar to those in our previous investigation. ${ }^{3}$ Other investigators have documented increases in $\mathrm{CI},{ }^{24,32}$ decreases in SVR, ${ }^{24,29,32}$ increases in shunt flow, $25,33,34$ and decreased incidence of

${ }^{*}$ References 3,5,6,12,19,22,24,28-30,32 
arrhythmias ${ }^{28}$ when methylprednisolone is used in this setting. Postoperative vasodilation, although having certain beneficial physiologic effects (decreased afterload and decreased left ventricular end-diastolic volume and pressure), may increase postoperative use of pharmacologic agents to support mean arterial pressure ${ }^{3}$ increase postoperative A-a oxygen gradient because of increased shunt fraction,,$^{2,3}$ or both. Indeed, after cardiac surgery, there is a significant correlation $(r=0.82)$ between shunt flow and A-a oxygen gradient. ${ }^{33}$

Whether administration of methylprednisolone before CPB attenuates pulmonary damage is also controversial. ${ }^{2,15,16,25,26}$ Some investigators document decreased neutrophil activation, ${ }^{16}$ decreased postoperative radiographic abnormalities, ${ }^{26}$ and improvement in postoperative oxygenation ${ }^{2}$ in patients who received methylprednisolone, whereas others reveal the drug does not prevent interleukin 8-mediated pulmonary neutrophil infiltration ${ }^{15}$ or postoperative oxygenation abnormalities..$^{25}$ As in our previous investgation, ${ }^{2}$ we again found that methylprednisolone was unable to prevent postoperative increases in A-a oxygen gradient and postoperative decreases in dynamic and static lung compliance.

The stimulus for this investigation was the unexpected findings of our previous investigation. ${ }^{2,3}$ Our group was the first to rigorously examine use of methylprednisolone in patients undergoing cardiac surgery and early extubation, and we found that the drug significantly prolonged extubation time when compared with placebo controls $(12.8 \pm 4.9$ hours vs $10.1 \pm 5.2$ hours, respectively; $P=.05){ }^{2,3}$ Using the same perioperative study protocol (with an additional group receiving the drug at half the original dose), we again found that methylprednisolone significantly prolonged extubation time. Because large doses of methylprednisolone may induce sodium and water retention, which may initiate pulmonary edema in susceptible patients, ${ }^{4}$ we also assessed perioperative fluid balance and weights. However, there was no difference between groups regarding these perioperative variables. Two previous observational studies further suggest that methylprednisolone prolongs extubation time in this setting. . $^{10,30}$ One found that patients who received $1.0 \mathrm{~g}$ of methylprednisolone immediately before CPB followed by 4 doses of dexamethasone ( $4.0 \mathrm{mg}$ each) every 6 hours after CPB had prolonged extubation times compared with control patients who did not receive steroids (13.1 \pm 2.3 hours vs $10.5 \pm 1.0$ hours, respectively), although the difference was not statistically significant. ${ }^{10}$ The other found that patients who received $30 \mathrm{mg} / \mathrm{kg}$ methylprednisolone after the induction of anesthesia required significantly prolonged respiratory support in
Table VII. Extubation times

\begin{tabular}{lc}
\hline All extubated patients & \\
Group NS, $\mathrm{h}(\mathrm{n}=27)$ & $7.0 \pm 5.1$ \\
Group 15MP, $\mathrm{h}(\mathrm{n}=28)$ & $14.9 \pm 34.7$ \\
Group 30MP, $\mathrm{h}(\mathrm{n}=30)$ & $16.3 \pm 38.7$ \\
Patients extubated within 24 $\mathrm{h}$ of ICU arrival & \\
Group NS, $\mathrm{h}(\mathrm{n}=27)$ & $7.0 \pm 5.0$ \\
Group 15MP, $\mathrm{h}(\mathrm{n}=27)$ & $8.4 \pm 5.4$ \\
Group 30MP, $\mathrm{h}(\mathrm{n}=29)$ & $9.3 \pm 5.0$ \\
Patients extubated within 12 $\mathrm{h}$ of ICU arrival & \\
Group NS, $\mathrm{h}(\mathrm{n}=25)$ & $5.7 \pm 2.3$ \\
Group 15MP, $\mathrm{h}(\mathrm{n}=21)$ & $5.9 \pm 2.2$ \\
Group 30MP, $\mathrm{h}(\mathrm{n}=24)$ & $7.5 \pm 2.7^{*}$ \\
\hline
\end{tabular}

$* P=.038$, group $30 \mathrm{MP}$ versus group NS.

the immediate postoperative period compared with historical controls $(85 \pm 181$ hours vs $27 \pm 16$ hours, respectively; $P=.05){ }^{30}$

Methylprednisolone is an attractive agent for potential suppression of the systemic inflammatory response associated with CPB because of its beneficial effects on neutrophil kinetics and function and because it represents one of the few therapeutic drugs that possess multi-inhibitory effects on numerous components of the inflammatory response. ${ }^{35}$ Potential anti-inflammatory effects of methylprednisolone, when used in this manner, include decreased complement activation, ${ }^{10,19,21}$ decreased interleukin 6 release, ${ }^{7,11,12}$ decreased interleukin 8 release, ${ }^{8-10,12}$ increased interleukin 10 release, ${ }^{8,9}$ decreased tumor necrosis factor release, ${ }^{9,11-13}$ and decreased neutrophil integrin CD11b up-regulation, ${ }^{13,14}$ among others. However, although results from animal models appear promising, definitive clinical benefits in human subjects have yet to be demonstrated. Our investigation shows that the drug confers no clinical benefit and may in fact be detrimental by delaying postoperative extubation (for undetermined reasons) and initiating postoperative hyperglycemia. Furthermore, methylprednisolone administration is not without other potential risks, which include gastritis, gastrointestinal bleeding, impaired wound healing, increased risk of infection, adverse psychiatric effects, avascular bone necrosis, and aggravation of ischemic brain injury (irrespective of hyperglycemia). ${ }^{36}$

In conclusion, this prospective randomized doubleblind placebo-controlled clinical study reveals that administration of methylprednisolone to patients undergoing elective $\mathrm{CABG}$ with $\mathrm{CPB}$ increases postoperative CI, decreases postoperative SVR, increases postoperative shunt flow, is unable to prevent postoperative pulmonary dysfunction (increased A-a oxygen gradient and decreased dynamic and static lung com- 
pliance) or postoperative weight gain, initiates postoperative hyperglycemia, and prolongs tracheal extubation time (for undetermined reasons). Thus, methylprednisolone, as used in this investigation, offers no clinical benefits to patients undergoing elective $\mathrm{CABG}$ with CPB and may in fact be detrimental by initiating postoperative hyperglycemia and possibly hindering early postoperative tracheal extubation.

Received for publication Aug 2, 2000; revisions requested Sept 27, 2000; revisions received Oct 2, 2000; accepted for publication Oct 16, 2000.

Address for reprints: Mark A. Chaney, MD, Department of Anesthesia and Critical Care, University of Chicago, 5841 South Maryland Ave, MC-4028, Chicago, IL 60637 (E-mail: mchaney@airway2.uchicago.edu).

\section{REFERENCES}

1. Asimakopoulos G, Smith PLC, Ratnatunga CP, Taylor KM. Lung injury and acute respiratory distress syndrome after cardiopulmonary bypass. Ann Thorac Surg 1999;68:1107-15.

2. Chaney MA, Nikolov MP, Blakeman B, Bakhos M, Slogoff S. Pulmonary effects of methylprednisolone in patients undergoing coronary artery bypass grafting and early tracheal extubation. Anesth Analg 1998;87:27-33.

3. Chaney MA, Nikolov MP, Blakeman BP, Bakhos M, Slogoff S. Hemodynamic effects of methylprednisolone in patients undergoing cardiac operation and early extubation. Ann Thorac Surg 1999;67:1006-11.

4. A-Methapred [package insert]. North Chicago: Abbott Laboratories; 1996.

5. Moses ML, Camishion RC, Tokunaga K, Pierucci L, Davies AL, Nealon TF. Effect of corticosteroid on the acidosis of prolonged cardiopulmonary bypass. J Surg Res 1966;6:354-60.

6. Thompson MA, Broadbent MP. Methylprednisolone prior to cardiopulmonary bypass. Anaesthesia 1980;35:345-53.

7. Diego RP, Mihalakakos PJ, Hexum TD, Hill GE. Methylprednisolone and full-dose aprotinin reduce reperfusion injury after cardiopulmonary bypass. J Cardiothorac Vasc Anesth 1997;11:29-31.

8. Tabardel Y, Duchateau J, Schmartz D, Marecaux G, Shahla M, Barvais L, et al. Corticosteroids increase blood interleukin-10 levels during cardiopulmonary bypass in men. Surgery 1996;119:76-80.

9. Wan S, DeSmet JM, Antoine M, Goldman M, Vincent JL, LeClerc JL. Steroid administration in heart and heart-lung transplantation: Is the timing adequate? Ann Thorac Surg 1996;61:674-8.

10. Engelman RM, Rousou JA, Flack JE, Deaton DW, Kalfin R, Das DK. Influence of steroids on complement and cytokine generation after cardiopulmonary bypass. Ann Thorac Surg 1995;60:801-4.

11. Hill GE, Snider S, Galbraith TA, Forst S, Robbins RA. Glucocorticoid reduction of bronchial epithelial inflammation during cardiopulmonary bypass. Am J Respir Crit Care Med 1995; 152:1791-5.

12. Teoh KHT, Bradley CA, Gauldie J, Burrows H. Steroid inhibition of cytokine-mediated vasodilation after warm heart surgery. Circulation 1995;92:II-347-53.

13. Hill GE, Alonso A, Spurzem JR, Stammers AH, Robbins RA. Aprotinin and methylprednisolone equally blunt cardiopulmonary bypass-induced inflammation in humans. $\mathrm{J}$ Thorac Cardiovasc Surg 1995;110:1658-62.

14. Hill GE, Alonso A, Thiele GM, Robbins RA. Glucocorticoids blunt neutrophil CD11b surface glycoprotein upregulation during cardiopulmonary bypass in humans. Anesth Analg 1994;79:23-7.

15. Jorens PG, De Jongh R, De Backer W, van Damme J, van Overveld F, Bossaert L, et al. Interleukin- 8 production in patients undergoing cardiopulmonary bypass: the influence of pretreatment with methylprednisolone. Am Rev Respir Dis 1993;148:890-5.

16. Jansen NJG, van Oeveren W, van Vliet M, Stoutenbeek CP, Eysman L, Wildevuur CRH. The role of different types of corticosteroids on the inflammatory mediators in cardiopulmonary bypass. Eur J Cardiothorac Surg 1991;5:211-7.

17. Fosse E, Mollnes TE, Osterud A, Aasen AO. Effects of methylprednisolone on complement activation and leukocyte counts during cardiopulmonary bypass. Scand J Thorac Cardiovasc Surg 1987;21:255-61.

18. Tennenberg SD, Bailey WW, Cotta LA, Brodt JK, Solomkin JS. The effects of methylprednisolone on complement-mediated neutrophil activation during cardiopulmonary bypass. Surgery 1986;100:134-42.

19. Cavarocchi NC, Pluth JR, Schaff HV, Orszulak TA, Homburger HA, Solis E, et al. Complement activation during cardiopulmonary bypass: comparison of bubble and membrane oxygenators. J Thorac Cardiovasc Surg 1986;91:252-8.

20. Boscoe MJ, Yewdall VMA, Thompson MA, Cameron JS. Complement activation during cardiopulmonary bypass: quantitative study of effects of methylprednisolone and pulsatile flow. BMJ 1983;287:1747-50.

21. Hammerschmidt DE, Stroncek DF, Bowers TK, Lammi-Keefe CJ, Kurth DM, Ozalins A, et al. Complement activation and neutropenia occurring during cardiopulmonary bypass. J Thorac Cardiovasc Surg 1981;81:370-7.

22. Toledo-Pereyra LH, Lin CY, Kundler H, Replogle RL. Steroids in heart surgery: a clinical double-blind and randomized study. Am Surg 1980;46:155-60.

23. Haslam PL, Townsend PJ, Branthwaite MA. Complement activation during cardiopulmonary bypass. Anaesthesia 1980;35:22-6.

24. Niazi Z, Flodin P, Joyce L, Smith J, Mauer H, Lillehei RC. Effects of glucocorticosteroids in patients undergoing coronary artery bypass surgery. Chest 1979;76:262-8.

25. Enderby DH, Boylett A, Parker DJ. Methylprednisolone and lung function after cardiopulmonary bypass. Thorax 1979;34:720-5.

26. Fecht DC, Magovern GJ, Park SB, Merkow LP, Dixon CM, Dosios $\mathrm{T}$, et al. Beneficial effects of methylprednisolone in patients on cardiopulmonary bypass. Circ Shock 1978;5:415-22.

27. Gnanadurai TV, Branthwaite MA, Colbeck JF, Welman E. Lysosomal enzyme release from the lungs after cardiopulmonary bypass. Anaesthesia 1978;33:227-31.

28. Rao G, King J, Ford W, King G. The effects of methylprednisolone on the complications of coronary artery surgery. Vasc Surg 1977;11:1-7.

29. Dietzman RH, Lunseth JB, Goott B, Berger EC. The use of methylprednisolone during cardiopulmonary bypass; a review of 427 cases. J Thorac Cardiovasc Surg 1975;69:870-3.

30. Coffin LH, Shinozaki T, DeMeules JE, Browdle DA, Deane RSD, Morgan JG. Ineffectiveness of methylprednisolone in the 
treatment of pulmonary dysfunction after cardiopulmonary bypass. Am J Surg 1975;130:555-9.

31. Wilson JW, Young WG, Miller BJ. Pulmonary cellular changes prevented or altered by methyl prednisolone sodium succinate. Fed Proc 1971;30:686.

32. Dietzman RH, Castaneda AR, Lillehei CW, Ersek RA, Motsay GJ, Lillehei RC. Corticosteroids as effective vasodilators in the treatment of low output syndrome. Chest 1970;57:440-53.

33. Hachenberg T, Tenling A, Nystrom SO, Tyden H, Hedenstierna G. Ventilation-perfusion inequality in patients undergoing cardiac surgery. Anesthesiology 1994;80:509-19.

\section{Appendix I. Hemodynamic standard equations}

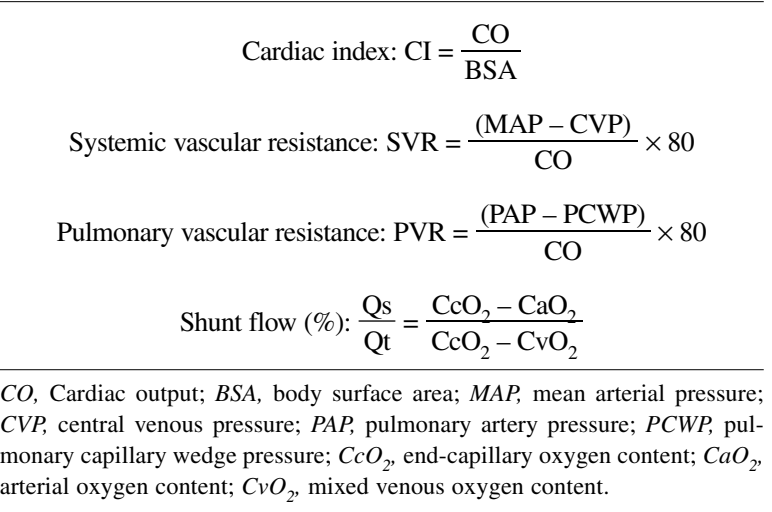

34. Hachenberg T, Tenling A, Rothen HU, Nystrom SO, Tyden H, Hedenstierna G. Thoracic intravascular and extravascular fluid volumes in cardiac surgical patients. Anesthesiology 1993;79:976-84.

35. Munck A, Guyre PM, Holbrook NJ. Physiological functions of glucocorticoids in stress and their relation to pharmacological actions. Endocr Rev 1984;5:25-44.

36. Tsubota S, Adachi N, Chen J, Yorozuya T, Nagaro T, Arai T. Dexamethasone changes brain monoamine metabolism and aggravates ischemic neuronal damage in rats. Anesthesiology 1999;90:515-23.

\section{Appendix II. Pulmonary standard equations}

Alveolar-arterial $\mathrm{O}_{2}$ gradient $(\mathrm{mm} \mathrm{Hg}): \mathrm{P}(\mathrm{A}-\mathrm{a}) \mathrm{O}_{2}=\mathrm{PAO}_{2}-\mathrm{PaO}_{2}$

$$
\begin{gathered}
\mathrm{PAO}_{2}=\mathrm{PIO}_{2}-\frac{\mathrm{PaCO}_{2}}{0.8} \\
\mathrm{PIO}_{2}=\mathrm{FIO}_{2} \times\left(\mathrm{P}_{\mathrm{B}}-47\right)
\end{gathered}
$$

Dynamic lung compliance $\left(\mathrm{mL} / \mathrm{cm} \mathrm{H}_{2} \mathrm{O}\right): \mathrm{Cd}=\frac{\text { Tidal volume }}{\mathrm{P} \text { peak }- \text { PEEP }}$

Static lung compliance $\left(\mathrm{mL} / \mathrm{cm} \mathrm{H}_{2} \mathrm{O}\right): \mathrm{Cs}=\frac{\text { Tidal volume }}{\mathrm{P} \text { plateau }-\mathrm{PEEP}}$

$\mathrm{PAO}_{2}$, Alveolar $\mathrm{PO}_{2}, \mathrm{PaO}_{2}$, arterial $\mathrm{Po}_{2} ; \mathrm{PaCO}_{2}$, arterial $\mathrm{PCO}_{2} ; 0.8$, assumed respiratory quotient; $P E E P$, positive end-expiratory pressure.

\section{Targeted}

The Journal of Thoracic and Cardiovascular Surgery gives you two tables of contents.

The condensed table of contents tells you at a glance what topics and authors are presented each month. The expanded table of contents gives you a brief abstract of each article. You select only those articles of most interest to you for further reading. 